\title{
An EM Scattering Algorithm for all Materials
}

\author{
A. Seagar ${ }^{1}$
}

\begin{abstract}
A simple iterative algorithm is presented for the calculation of the electromagnetic radiation scattered from objects of arbitrary shape made from either lossless or lossy dielectrics or conductors. Convergence of the algorithm is tested on materials of all such types. It is found that the algorithm converges for all materials; faster for lossy ones.
\end{abstract}

\section{INTRODUCTION}

Chantaveerod [1] reports iterative algorithms for the calculation of electromagnetic scattering from objects which are either perfect conductors or lossless dielectrics. The algorithms are formulated using a Cauchy integral constructed around the (first order) Dirac differential operator in three (or indeed any number of) dimensions cast in the framework of Clifford algebra. For want of any other name, here we call this approach the Clifford-CauchyDirac (CCD) formulation.

The CCD formulation has certain characteristics which distinguish it from conventional integral equation approaches. The use of Clifford algebra provides a framework in which the electromagnetic field is represented as a whole entity rather than two dissociated components. In common with combined field integral equation (CFIE) approaches this avoids the pitfalls of treating electric and magnetic fields in isolation $[2,3]$. However, there the similarity ceases.

Use of the Cauchy integral and Dirac operator as a pair, rather than Helmholtz operator with the associated integrals based on Greens functions, establishes the method as a differential or integral system of a first rather than of a second order. The Greens function and its derivative are replaced by the Cauchy kernel (without derivatives), and the double integrals and somewhat treacherous second order singularities are replaced by single integrals and singularities of the first order. The combination of milder singularities and whole field approach admits a single numerical solution which encompass the entire range of physical parameters: frequency, material properties, and shape.

The CFIE formulation by comparison is different. In spite of its apparent generality, it requires a

\footnotetext{
${ }^{1}$ School of Engineering, Griffith University, Gold Coast, Queensland, Australia, e-mail: dr_andrew_seagar@ieee.org.

${ }^{2}$ School of Engineering and Resources, Walailak University, Nakon Si Thammarat, Thailand, e-mail: cyodchai@gmail.com.
}

\section{A. Chantaveerod ${ }^{2}$}

variety of special cases to produce accurate results in all situations. Different formulations are used for different types of materials; conductors [4] and dielectrics [5,6] singly or together $[2,3,7,8]$; and even for a single type of material accuracy can be quite variable [8-11] over a range of values of material properties.

This makes the CCD approach and the CFIE methods quite different, both in terms of formulation and performance.

\section{FORMULATION}

Figure 1 shows an object $\Omega^{+}$of arbitrary shape in one or more dimensions, with uniform electrical conductivity $\sigma_{+}$, permittivity $\epsilon_{+}$and permeability $\mu_{+}$. The object is embedded in a region of free space $\Omega^{-}$with conductivity $\sigma_{-}=0$, permittivity $\epsilon_{-}=\epsilon_{0}$ and permeability $\mu_{-}=\mu_{0}$.

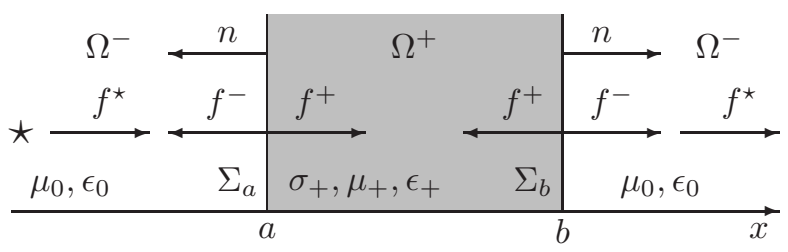

Figure 1: Radiation from source $\star$ is transmitted and reflected at surface $\Sigma$ of scatterer $\Omega^{+}$embedded in free space $\Omega^{-}$.

A linearly polarised plane wave of electromagnetic radiation $f^{\star}$ from a distant source $\star$ strikes the surface $\Sigma=\Sigma_{a} \cup \Sigma_{b}$ of the object $\Omega^{+}$. Part of the wave $f^{+}$penetrates and is admitted into the object, and part of the wave $f^{-}$fails to penetrate and is scattered away from the object. The total field measured in free space $\Omega^{-}$is constructed from the sum of the incident plane wave $f^{\star}$ and the scattered (or reflected) wave $f^{-}$. Inside the object the total field is constructed from the admitted (or transmitted) wave $f^{+}$alone.

\section{SOLUTION}

The solution for the fields outside and inside the object involves two steps. The first step is the calculation of $f^{+}$and $f^{-}$on the surface $\Sigma$ in whole field Clifford form (see table I in [12]). The second step is the extension of those fields away from the bound- 
ary using the Cauchy extension formula $[13,14]$ :

$$
C f(\mathbf{r} \notin \Sigma)=\int_{\Sigma} E_{k}(\mathbf{s}-\mathbf{r}) n(\mathbf{s}) f(\mathbf{s}) d \sigma(\mathbf{s})
$$

where $n(\mathbf{s})$ is the normal to the boundary $\Sigma, d \sigma(\mathbf{s})$ is the elemental measure of boundary, and $E_{k}$ is the Cauchy kernel. For three spatial dimensions [12]:

$$
E_{k}(\mathbf{r})=-\frac{1}{4 \pi r^{2}}\left(\hat{\mathbf{r}}+i k r\left(\hat{\mathbf{r}}-i e_{0}\right)\right) e^{-i k r}
$$

and for one spatial dimension:

$$
E_{k}(\mathbf{r})=-\frac{1}{2}\left(\hat{\mathbf{r}}-i e_{0}\right) e^{-i k r}
$$

When calculating the Cauchy extension $C=C^{ \pm}$ into the two regions $\Omega^{-}$and $\Omega^{+}$the wavenumber $k=\omega / c$ in the Cauchy kernel is constructed from the material properties in the corresponding region.

The Cauchy extension is the easiest step. All variables on the right hand side of equation (1) are known and are simply combined to produce the result on the left hand side. No kind of inversion is necessary. The hardest step is calculating the fields $f^{ \pm}$on the surface. Here that step is effected by iteration using the two simple formula:

$$
\begin{aligned}
& f_{2 n}^{+}= \\
& P^{++}\left\{\frac{1}{2}(I+R Q) f_{2 n-1}^{-}-g+\frac{1}{2}(I+R Q) f_{2 n-2}^{+}\right\}
\end{aligned}
$$

and:

$$
\begin{aligned}
& f_{2 n+1}^{-}= \\
& P^{--}\left\{\frac{1}{2}(I-R Q) f_{2 n}^{+}+g+\frac{1}{2}(I-R Q) f_{2 n-1}^{-}\right\}
\end{aligned}
$$

Each of the fields is updated iteratively, $n=$ $1,2,3 \ldots$, from earlier estimates of both fields. The iteration can be started with a first estimate for the fields $f_{0}^{+}=f_{1}^{-}=0$.

In equations (4) and (5) the operators $P^{++}$and $P^{--}$are Hardy projection operators:

$$
\left\{\begin{array}{l}
P^{++}=\frac{1}{2}\left(I+C_{\Sigma}^{+}\right) \\
P^{--}=\frac{1}{2}\left(I-C_{\Sigma}^{-}\right)
\end{array}\right.
$$

defined in terms of the Cauchy integral [14]:

$$
C_{\Sigma} f(\mathbf{r} \in \mathbf{\Sigma})=2 \text { p.v. } \int_{\Sigma} E_{k}(\mathbf{s}-\mathbf{r}) n(\mathbf{s}) f(\mathbf{s}) d \sigma(\mathbf{s})
$$

where "p.v." indicates that the Cauchy principal value of the integral is taken to properly account for the singularity in the Cauchy kernel $E_{k}$ at $\mathbf{s}=\mathbf{r}$. Note that the Cauchy extension formula (1) and the Cauchy integral equation (7) differ. The Cauchy extension has no singularity and produces fields away from the surface. The Cauchy integral contains an integrable singularity and produces fields on the surface.
For Hardy projections $P^{ \pm \pm}$and Cauchy integrals $C_{\Sigma}=C_{\Sigma}^{ \pm}$associated with the regions $\Omega^{-}$and $\Omega^{+}$the wavenumber $k$ in the Cauchy kernel is calculated from the material properties in the corresponding region $\Omega^{ \pm}$.

The Hardy projections serve to enforce Maxwell's equations, through their formulation from the Dirac operator. That operator is effectively a modern form of the quaternion operator used first by Maxwell, discarded by Gibbs and Heaviside, and rediscovered by Dirac in a different context.

The boundary conditions are enforced by the value:

$$
g=-\frac{1}{2}(I+R Q) f^{\star}
$$

calculated from the incident field at the surface of the object. This value serves as data for any particular problem.

The terms $I \pm R Q$ are linear operators which involve both the reflection $(Q)$ and rescaling $(R)$ of fields. The reflection operator $Q$ calculates the image of any field as reflected in the surface of the object. The rescaling operator $R$ applies separate scale factors to the space-like (magnetic) and timelike (electric) components of the field according to the values of the material properties on either side of the surface, in order to honour the boundary conditions on field intensities and flux densities. For full details see [15].

\section{APPLICATION}

The algorithm of equations (4) and (5) applies unmodified in either one or three dimensions simply by taking the corresponding value of the Cauchy kernel from equations (2) and (3). In both cases convergence is governed by the Hardy projections and the linear mappings $I \pm R Q$. The algorithm exhibits the general nature of the method of alternating projections, but with extra steps inserted.

For methods involving alternating projections convergence is governed by the particular properties of the projections themselves [16], and may or may not occur. In [1] Chantaveerod demonstrates for perfect conductors that his iterative method does properly converge when the three dimensional Cauchy kernel is used. Here a much more thorough examination is performed for materials of all types with the more general algorithm of equations (4) and (5). The examination is restricted to the one dimensional Cauchy kernel.

\section{TEST CASES}

Convergence of the algorithm in terms of the RMS value of the difference $\Delta g$ between the actual data 


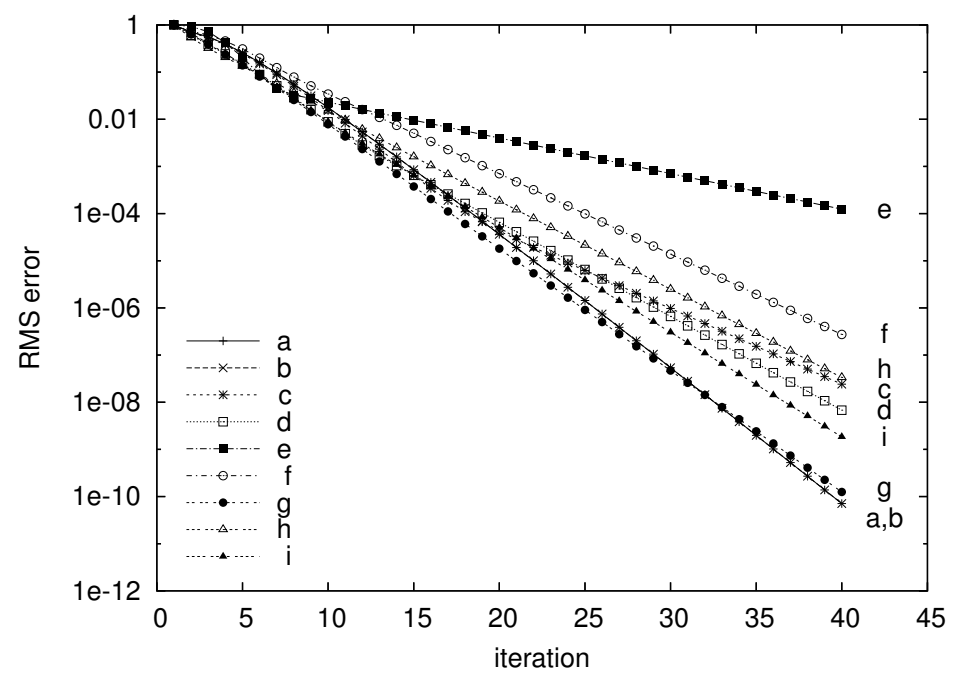

Figure 2: Reduction of error during convergence of solution for materials listed in table 1.

$g$ and that given by the current estimates of the fields:

$$
\Delta g_{n}=\left\|\frac{1}{2}(I+R Q) f_{2 n+1}^{-}-\frac{1}{2}(I-R Q) f_{2 n}^{+}-g\right\|
$$

has been examined for the materials listed in table 1; characterised electrically at ambient temperature by the lossless component of the permittivity $\epsilon$ and by the lossy component in terms of the loss tangent, $\tan \delta$, and conductivity $\sigma$.

\begin{tabular}{|l|c|c|c|}
\hline material & $\begin{array}{c}\text { permittivity } \\
\epsilon / \mathrm{Fm}^{-1}\end{array}$ & $\begin{array}{c}\text { loss tangent } \\
\tan \delta\end{array}$ & $\begin{array}{c}\text { conductivity } \\
\sigma / \Omega^{-1} \mathrm{~m}^{-1}\end{array}$ \\
\hline a copper & & & $5.83 \times 10^{7}$ \\
\hline b antimony & & & $2.56 \times 10^{6}$ \\
\hline c sea water & $81 \epsilon_{0}$ & & 5.3 \\
\hline d silicon & $11.7 \epsilon_{0}$ & & $4.25 \times 10^{-4}$ \\
\hline e water & $80.2 \epsilon_{0}$ & & $5.5 \times 10^{-6}$ \\
\hline f ethanol & $25.3 \epsilon_{0}$ & & $1.35 \times 10^{-7}$ \\
\hline g silica glass & $3.8 \epsilon_{0}$ & 0.00038 & $2.0 \times 10^{-8}$ \\
\hline h sapphire & $11.5 \epsilon_{0}$ & 0.0001 & $1 \times 10^{-9}$ \\
\hline i PVC & $3.0 \epsilon_{0}$ & 0.015 & $1.0 \times 10^{-13}$ \\
\hline
\end{tabular}

Table 1: Test cases (a) metal, (b) semi-metal, (c) sea water, (d) semiconductor, (e) pure water, (f) organic, (g) glass, (h) ceramic, (i) polymer.

The values in the table are the most extreme (away from vacuum) found from various sources, in order to test the algorithm over the full range expected of material properties. The highest values of permittivity are typically found at lower frequencies. Samples of water or semiconductors which are not pure can have values quite different from those shown. The tests were performed for a one dimensional scatterer with thickness $b-a$ in figure 1 equal to five wavelengths in free space, at an angular frequency $\omega=2 \pi \times 10^{9} \mathrm{~Hz}$.

\section{RESULTS}

Figure 2 shows the reduction in error $\Delta g_{n}$ of the solution for the materials listed in table 1 . The convergence is linear, with different rates of convergence (i.e. the slopes $\Delta g_{n+1} / \Delta g_{n}$ ) for the different materials listed in table 2 . Lower values represent a faster convergence.

\begin{tabular}{|c|ccccccccc|}
\cline { 2 - 9 } \multicolumn{1}{c|}{} & \multicolumn{1}{c|}{ m } & b & c & d & e & f & g & h & i \\
\hline rate & 0.51 & 0.51 & 0.69 & 0.63 & 0.84 & 0.67 & 0.55 & 0.64 & 0.60 \\
\hline
\end{tabular}

Table 2: Rates of convergence for materials listed in table 1 .

Comparing the results in table 2 to the values in table 1 shows that the rate of convergence is related to both the permittivity of the material (in contrast to its surroundings) and the lossy component for the material, from both free and bound charges. Convergence is typically fastest when the permittivity is low and the loss is high, as exhibited here by the cases of good conductors, i.e. metals and semi-metals. Convergence is typically slowest when the permittivity is high and the losses are low; most notably here for polar liquids: pure water and ethanol.

In absolute terms most materials exhibit convergence to within five decimal places in 30 iterations. The exceptions are ethanol with an error of $0.0014 \%$ after 30 iterations, and pure water for which the error remains $0.012 \%$ after 40 iterations.

The general trend is consistent with results of Chantaveerod [1] for a conducting cube in three dimensions. The implication is that the more general iterative method of equations (4) and (5) converges equally well for other materials in three dimensions. 


\section{CONCLUSIONS}

The new iterative algorithm presented here for solving electromagnetic scattering problems falls into that class known as the method of alternating projections. The projections involved are Hardy projections, which are constructed from Cauchy integrals in such a way that they enforce Maxwell's equations in each region with different material properties. The method is shown experimentally to converge to within five decimal places for most types of materials in about 30 iterations.

\section{References}

[1] A. Chantaveerod and A. Seagar, "Iterative solutions for electromagnetic fields at perfectly reflective and transmissive interfaces using Clifford algebra and the multi-dimensional Cauchy integral," IEEE Transactions on Antennas and Propagation, vol. 57, no. 11, pp. 3489-3499, Nov. 2009.

[2] B. Jung and T. Sakar, "Analysis of scattering from arbitrarily shaped 3-D conducting/dielectric composite objects using a combined field integral equation," Journal of Electromagnetic Waves and Application, vol. 18, no. 6, pp. 729-743, 2004.

[3] B. Jung, T. Sakar, and M. Salazar-Palma, "Combined field integral equation for the analysis of scattering from 3D conducting bodies coated with a dielectric material," Microwave and Optical Technology Letters, vol. 40, no. 6, pp. 511-516, Mar. 2004.

[4] S. Rao, D. Wilton, and A. Glisson, "Electromagnetic scattering by surfaces of arbitrary shape," IEEE Transactions on Antennas and Propagation, vol. 30, no. 3, pp. 409-418, May 1982.

[5] S. Rao and D. Wilton, "E-field, H-field, and combined field solution for arbitrarily shaped three-dimensional dielectric bodies," Electromagnetics, vol. 10, no. 4, pp. 407-421, 1990.

[6] P. Ylä-Oijala and M. Taskinen, "Calculation of CFIE impedance matrix elecments with RWG and $n \times$ RWG functions," IEEE Transactions on Antennas and Propagation, vol. 51, no. 8, pp. 1837-1846, Aug. 2003.

[7] — , "Application of combined field integral equation for electromagnetic scattering by dielectric and composite objects," IEEE Transactions on Antennas and Propagation, vol. 53, no. 3, pp. 1168-1173, Mar. 2005.
[8] P. Ylä-Oijala, "Numerical analysis of combined field integral equation formulations for electromagnetic scattering by dielectric and composite objects," Progress in Electromagnetics Research $C$, vol. 3, pp. 19-43, 2008.

[9] T. Lloyd, J. Song, and M. Yang, "Numerical study of surface integral formulations for lowcontrast objects," IEEE Antennas and Wireless Propagation Letters, vol. 4, no. 1, pp. 482485, 2005.

[10] Ö. Ergül and L. Gürel, "Fast and accurate solutions of scattering problems involving dielectric objects with moderate and low contrasts," in Computational Electromagnetics Workshop, 2007, Zmir, Aug. 30-31 2007, pp. 59-64.

[11] — , "Accurate solutions of scattering problems involving low-contrast dielectric objects with surface integral equations," in Antennas and Propagation (EuCAP 2007), The Second European Conference on, Edinburgh, Nov. 1116 2007, pp. 1-6.

[12] A. Seagar, "Calculation of electromagnetic fields in three dimensions using the Cauchy integral," IEEE Transactions on Magnetics, vol. 48, no. 2, pp. 175-178, Feb. 2012.

[13] A. McIntosh and M. Mitrea, "Clifford algebras and Maxwell's equations in Lipschitz domains," Mathematical Methods in the Applied Sciences, vol. 22, no. 18, pp. 1599-1620, Dec. 1999.

[14] A. Axelsson, R. Grognard, J. Hogan, and A. McIntosh, "Harmonic analysis of Dirac operators on Lipschitz domains," in Clifford Analysis and its Applications, ser. NATO Science Series II, Mathematics Physics and Chemistry, F. Brackx, Ed., vol. 25. Dordrecht: Kluwer, 2001, pp. 231-246.

[15] A. Seagar, Application of Geometric Algebra to Electromagnetic Scattering, in preparation.

[16] C. Badea, S. Grivaux, and V. Müller, "The rate of convergence in the method of alternating projections," St. Petersburg Mathematical Journal, vol. 23, no. 3, pp. 413-434, 2012. 